\title{
Severe Excessive Dynamic Airway Collapse in a Patient With Primary Sjögren's Syndrome
}

\author{
Sophie Ismael MD, Delphine Wermert MD, Kim-Diep Dang-Tran MD, Marion Venot MD, \\ Jean-Yves Fagon MD, and Jean-Luc Diehl MD
}

\begin{abstract}
Airway and cystic lung diseases can be observed in patients with Sjögren's syndrome. We report a case of such a patient suffering from respiratory failure due to recurrent episodes of right pneumothorax, requiring invasive mechanical ventilation. Despite thoracic drainage and adequate pneumothorax management, the patient could not be weaned from the ventilator. Fiberoptic bronchoscopy revealed severe central excessive dynamic airway collapse of the lower part of the trachea and proximal bronchi. The severity of airway collapse was maximal at the intermediate bronchus level, with a near-complete obstruction during expiration. Inspiratory and expiratory computed tomography studies confirmed the fiberoptic findings and suggested a possible expiratory posterior compression of the intermediate bronchus by parenchymal lung cysts. Stenting was considered, but the patient died from ventilator-associated pneumonia before the procedure could be performed. This case is the first description of severe central excessive dynamic airway collapse in a patient with primary Sjögren's syndrome complicated by diffuse airway and cystic lung disease. Key words: respiratory failure; expiratory dynamic airway collapse; Sjögren's syndrome; bronchoscopy; lung cysts; inspiratory and expiratory ct. [Respir Care 2014;59(10):e156-e159. (C) 2014 Daedalus Enterprises]
\end{abstract}

\section{Introduction}

Primary Sjögren's syndrome (PSS) is one of the most common autoimmune diseases. Its prevalence, second only to rheumatoid disease, is estimated in up to $4 \%$ of the population. ${ }^{1-4}$ PSS is mainly characterized by a sicca syn-

\footnotetext{
Drs Ismael, Venot, Fagon, and Diehl are affiliated with the Service de Réanimation Médicale, Hôpital Européen Georges Pompidou, Assistance Publique-Hopitaux de Paris; Dr Welmert is affiliated with the Service de Pneumologie, Hôpital Européen Georges Pompidou, Assistance PubliqueHopitaux de Paris; Dr Dang-Tran is affiliated with the Service de Radiologie, Hôpital Européen Georges Pompidou, Assistance PubliqueHopitaux de Paris, 20 rue Leblanc; Dr Diehl is also affiliated with the Institut National de la Santé et de la Recherche Médicale U765, Faculté de Pharmacie and Faculté de Médecine, Université Paris Descartes, Sorbonne Paris Cité, Paris, France.
}

The authors have disclosed no conflicts of interest.

Correspondence: Jean-Luc Diehl MD, Service de Réanimation Médicale, Hôpital Européen Georges Pompidou, Assistance Publique-Hopitaux de Paris, 20 rue Leblanc, 75015 Paris, France. E-mail: jldiehl@invivo.edu.

DOI: $10.4187 /$ respcare.02929 drome (persistent symptoms of dryness of eyes and mouth), which can be associated with other systemic manifestations. Clinical pulmonary involvement can affect up to $22 \%$ of PSS patients and is associated with reduced quality of life and an increase in mortality. ${ }^{4}$ There is a broad spectrum of pulmonary manifestations, including airway diseases (mainly lymphocytic bronchial inflammation and xerotrachea) and interstitial diseases (mainly nonspecific interstitial pneumonia, usual interstitial pneumonia, and organizing pneumonia). ${ }^{5-7}$ Pulmonary cysts have also been increasingly described, with prevalence up to $46 \% .^{5,7-13}$

We report here the case of a 57-y-old woman suffering from severe respiratory insufficiency related to lung involvement of PSS, mainly characterized by diffuse airway and cystic lung diseases. A severe excessive dynamic airway collapse (EDAC) of the central airways was observed, which was maximal at the intermediate bronchus level. EDAC was probably secondary to a PSS-related airway disease, with a possible accessory role of posterior compression exerted by the cysts in some bronchial areas. To the best of our knowledge, such a feature has never been described in PSS patients. This case stands as an example of severe respiratory manifestations of PSS and raises some important diagnostic and therapeutic questions. 


\section{Case Report}

A 57-y-old woman with a longstanding history of PSS was referred to our ICU for severe respiratory distress related to a right pneumothorax. PSS had been diagnosed $25 \mathrm{y}$ before referral. At the time of referral, the patient suffered from a sicca syndrome, polyarthralgias, and severe pulmonary involvement. A labial salivary gland biopsy showed a sialiadenitis with a focus score $>1$. At this time, computed tomography (CT) scan studies displayed large areas of emphysema and cysts predominantly in the right lower lobe. A low-dosage systemic corticosteroid regimen had been administered since diagnosis of the PSS.

Results of pulmonary function testing performed $5 \mathrm{y}$ before admission were as follows: FVC $1.52 \mathrm{~L}(54 \%$ theoretical value), expiratory reserve volume $0.11 \mathrm{~L}(12 \%$ theoretical value), inspiratory reserve volume $0.81 \mathrm{~L}, \mathrm{FEV}_{1}$ $0.85 \mathrm{~L}$ ( $35 \%$ theoretical value), and residual volume 5.01 L (287\% theoretical value). Six months before admission, arterial blood gas results while breathing room air were as follows: $\mathrm{pH} 7.44, \mathrm{P}_{\mathrm{aO}_{2}} 63 \mathrm{~mm} \mathrm{Hg}$, and $\mathrm{P}_{\mathrm{aCO}_{2}} 46 \mathrm{~mm} \mathrm{Hg}$. Other biological explorations showed the presence of polyclonal hypergammaglobulinemia and anti-SSA and anti-SSB auto-antibodies. Rheumatoid factor and anti-cyclic citrullinated peptide auto-antibodies were not detected. A CT scan study displayed a frank aggravation, with large bilateral cysts. Alpha- 1 antitrypsin was within the normal range $(1.55 \mathrm{~g} / \mathrm{L})$. There was no evidence for lymphangiomyomatosis or histiocytosis. The patient did not suffer from renal insufficiency. She lived alone, had a son, and had no difficulty with activities of daily living until the 6 months preceding ICU admission.

During this period, she had been hospitalized twice because of recurrent right pneumothorax requiring thoracic drainage. She also complained of a worsening dyspnea. The systemic corticoid regimen (prednisone, $12 \mathrm{mg} / \mathrm{d}$ ) was continued, but with no adjunction of any other immunosuppressive agent. The patient was finally hospitalized in another institution for respiratory distress secondary to an acute bacterial pneumonitis, and referred to our ICU $6 \mathrm{~d}$ later because of hypercapnic respiratory failure requiring invasive mechanical ventilation. A chest CT study revealed diffuse pulmonary parenchymal cysts and a recurrent apical right pneumothorax, with a favorable course after chest tube insertion. The ventilator settings were as follows: ventilator-controlled continuous mandatory ventilation mode, tidal volume $8 \mathrm{~mL} / \mathrm{kg}$, breathing frequency 12 breaths/min, applied PEEP $5 \mathrm{~cm} \mathrm{H}_{2} \mathrm{O}$, with the aim to minimize the level of dynamic hyperinflation. No clinical, biological, or CT evidence was found for systemic amyloidosis or an associated lymphoproliferative disorder. Persistent weaning difficulties were observed, as well as recurrent episodes of ventilator-associated pneumonia. Fiberoptic bronchoscopy-obtained bronchoalveolar lavage


Fig. 1. Images from a video-bronchoscopic examination of the intermediate bronchus during inspiration $(A)$ and expiration $(B)$ under controlled mechanical ventilation. Severe excessive dynamic airway collapse was evident.

was performed as standard ventilator-associated pneumonia diagnostic procedure in our institution. ${ }^{14}$ Bronchoscopies suggested an EDAC of proximal bronchi and lower part of the trachea, which was confirmed by a video-bronchoscopic examination. The severity of the airway collapse was maximal at the intermediate bronchus level, with a near complete obstruction during expiration (Fig. 1). Cartilaginous tracheal and bronchial structures were visually judged as normal. Inspiratory and expiratory CT analysis confirmed the fiberoptic bronchoscopy findings and suggested a possible expiratory posterior compression of the intermediate bronchus by parenchymal lung cysts (Fig. 2). We applied the multidimensional FEMOS classification system for patients with expiratory central airway collapse proposed by Murgu and Colt, ${ }^{15}$ and found the following results:

- Functional status: 4

- Extent of abnormality: 4

- Morphology: EDAC

- Origin: secondary

- Severity of airway collapse: 4 


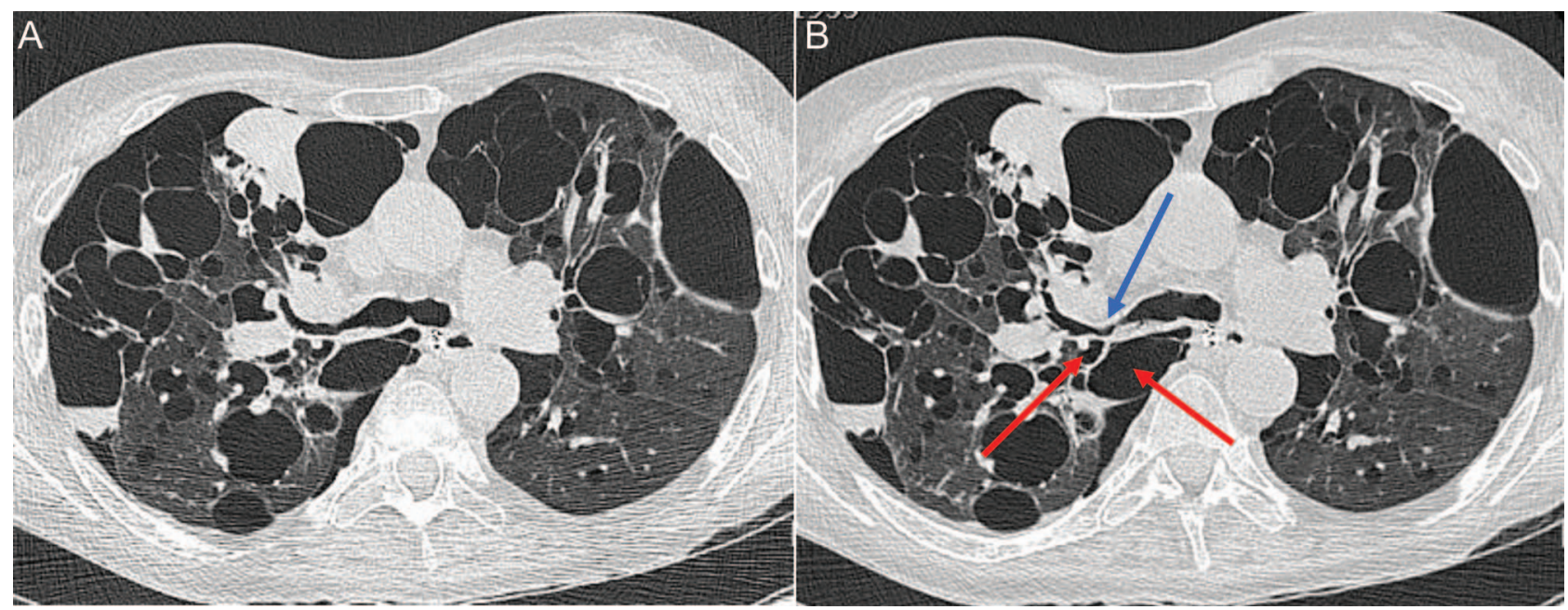

Fig. 2. Multidetector CT scans under controlled mechanical ventilation during an end-inspiratory pause (A) and during an end-expiratory pause (B) confirming severe excessive dynamic airway collapse of the intermediate bronchus (blue arrow). Cysts localized near to the posterior part of the bronchus demonstrated some level of air trapping during expiration (red arrows), which probably contributed to the magnitude of the bronchus collapse.

Even if the FEMOS classification is presented as an aid to objectively stratify patients, it suggested in the present case the need for therapeutic interventions such as stent insertion and/or reinforcement of immunosuppressive treatment, with the aim to wean the patient from mechanical ventilation. ${ }^{15}$ Stenting under rigid bronchoscopy was therefore considered, but the patient died from recurrent ventilator-associated pneumonia before the procedure could be performed. An immediate stent implantation under flexible bronchoscopy could also have been considered, but such a method is actually not currently available in our institution.

\section{Discussion}

We report the first case of severe proximal EDAC in a patient suffering from severe PSS-related pulmonary involvement. The patient fulfilled the 2002 American-European Consensus Group criteria for PSS. ${ }^{16}$ While clinical pulmonary involvement can affect up to $22 \%$ of PSS patients, ${ }^{4}$ pulmonary cysts have also been increasingly described, with prevalence up to $46 \% .^{5,7-13}$ Using high-resolution CT studies, Lohrmann et al ${ }^{5}$ reported in 24 PSS patients a high prevalence (79\%) of pathological findings, with almost half of the patients displaying thin-walled cysts.

Kobayashi et $\mathrm{al}^{8}$ and Bonner et al ${ }^{17}$ were the first to describe cases of bullae formation or lung cysts in relation to Sjögren's syndrome, hypothesizing that lung cysts could result from airway narrowing by peribronchiolar lymphoid cellular infiltration and/or amyloid deposition, with consequent air trapping. Such a view has been widely shared, even if a correlation between cystic lesions and airway obstruction was not always found. ${ }^{11}$ In fact, the exact mech- anism of lung cyst formation is still a matter of debate. The main advocated mechanism is therefore a partial airway obstruction, either caused by a peribronchial lymphoid cellular infiltration, as seen in lymphocytic interstitial pneumonia, or caused by an amyloid deposition, as seen in multinodular amyloidosis. These 2 conditions are known to be associated with Sjögren's syndrome. In our case, there was no argument for a multinodular amyloidosis.

EDAC was evident in our patient, according to the definition proposed by Kalra et al, ${ }^{18}$ ie, a pathological collapse and narrowing of the airway lumen by more than $50 \%$, which is entirely due to the laxity of the posterior wall membrane with structurally intact airway cartilage. As suggested by Kalra et al, ${ }^{18}$ EDAC could have been suspected in our patient by difficulties in weaning from mechanical ventilation and was evident by fiberoptic bronchoscopy with recording of short clips. CT studies performed during controlled mechanical ventilation with endinspiratory and end-expiratory pauses confirmed severe EDAC, which was maximal at intermediate bronchus level. Moreover, CT studies suggested some level of air trapping, particularly affecting cysts localized near to the posterior wall of the right main bronchus and of the intermediate bronchus. Therefore, we can hypothesize that the compression exerted by the cyst could have explained in part the higher severity of the right bronchus system collapse, as compared with the left one. Inspiratory and expiratory high-resolution $\mathrm{CT}$ studies were previously used by Meyer et $\mathrm{al}^{9}$ to demonstrate air trapping in a patient with secondary Sjögren's syndrome, but without mention of airway collapse. A dynamic expiratory CT study could have displayed more precise information, but was ruled out for our patient, because of the difficulties related to the 
coupling of mechanical ventilation and CT acquisitions and because of the already available results of the bronchoscopic examination. ${ }^{19}$ Therefore, one of the main objectives of the CT study in our case was to determine the degree of compression exerted by the cysts.

We applied in our patient the FEMOS classification system, proposed by Murgu and colleagues ${ }^{15,20}$ to help define the need for therapeutic interventions such as stent insertion and/or reinforcement of immunosuppressive treatment for PSS, with the aim to wean the patient from mechanical ventilation, and we found it to be of value for this purpose.

In conclusion, we report a patient with PSS and severe pulmonary involvement characterized by severe EDAC, mainly in relation to PSS bronchial disease, and cystic lung disease. Despite an unfavorable outcome, this case helps to discuss diagnostic and therapeutic strategies in such patients.

\section{REFERENCES}

1. Thomas E, Hay EM, Hajeer A, Silman AJ. Sjögren's syndrome: a community-based study of prevalence and impact. Br J Rheumatol 1998;37(10):1069-1076.

2. Pillemer SR, Matteson EL, Jacobsson LT, Martens PB, Melton LJ 3rd, O'Fallon WM, Fox PC. Incidence of physician-diagnosed primary Sjögren's syndrome in residents of Olmsted county, Minnesota. Mayo Clin Proc 2001;76(6):593-599.

3. Haugen AJ, Peen E, Hultén B, Johannessen AC, Brun JG, Halse AK, Haga HJ. Estimation of the prevalence of primary Sjögren's syndrome in two age-different community-based populations using two sets of classification criteria: the Hordaland Health Study. Scand J Rheumatol 2008;37(1):30-34.

4. Palm O, Garen T, Berge Enger T, Jensen JL, Lund MB, Aaløkken TM, Gran JT. Clinical pulmonary involvement in primary Sjögren's syndrome: prevalence, quality of life and mortality - a retrospective study based on registry data. Rheumatology 2013;52(1):173-179.

5. Lohrmann C, Uhl M, Warnatz K, Ghanem N, Kotter E, Schaefer O, Langer M. High-resolution CT imaging of the lung for patients with primary Sjögren's syndrome. Eur J Radiol 2004;52(2):137-143.

6. Lazor R. Lung involvement in Sjögren's syndrome: interstitium, airways, or both? Respiration 2009;78(4):375-376.
7. Watanabe M, Naniwa T, Hara M, Arakawa T, Maeda T. Pulmonary manifestations in Sjögren's syndrome: correlation analysis between chest computed tomographic findings and clinical subsets with poor prognosis in 80 patients. J Rheumatol 2010;37(2):365-373.

8. Kobayashi H, Matsuoka R, Kitamura S, Tsunoda N, Saito K. Sjögren's syndrome with multiple bullae and pulmonary nodular amyloidosis. Chest 1988;94(2):438-440.

9. Meyer CA, Pina JS, Taillon D, Godwin JD. Inspiratory and expiratory high-resolution CT findings in a patient with Sjögren's syndrome and cystic lung disease. Am J Roentgenol 1997;168(1):101103.

10. Koyama M, Johkoh T, Honda O, Mihara N, Kozuka T, Tomiyama N, et al. Pulmonary involvement in primary Sjögren's syndrome. Spectrum of pulmonary abnormalities and computed tomography findings in 60 patients. J Thor Imaging 2001;16(4):290-296.

11. Hubscher O, Re R, Iotti R. Cystic lung disease in Sjögren's syndrome. J Rheumatol 2002;29(10):2235-2236.

12. Jagirdar J, Chikkamuniyappa S, Sirohi D, McCarthy MJ, Peters JI. Cystic lung lesions in Sjogren syndrome: analysis of lymphocyte subsets in tissue with clinico-radiologic-pathologic correlation. Ann Diagn Pathol 2013;17(1):113-116.

13. Baqir M, Kluka EM, Aubry MC, Hartman TE, Yi ES, Bauer PR, Ryu JH. Amyloid-associated cystic lung disease in primary Sjögren's syndrome. Respir Med 2013;107(4):616-621.

14. Chastre J, Fagon JY. Ventilator-associated pneumonia. Am J Respir Crit Care Med 2002;165(7):867-903.

15. Murgu SD, Colt HG. Description of a multidimensional classification system for patients with expiratory central airway collapse. Respirology 2007;12(4):543-550.

16. Vitali C, Bombardieri S, Jonsson R, Moutsopoulos HM, Alexander EL, Carsons SE, et al. Classification criteria for Sjögren's syndrome: a revised version of the European criteria proposed by the AmericanEuropean Consensus Group. Ann Rheum Dis 2002;61(6):554-558.

17. Bonner H, Ennis RS, Geelhoed GW, Tarpley TM. Lymphoid infiltration and amyloidosis of lung in Sjögren's syndrome. Arch Pathol 1973;95(1):42-44.

18. Kalra A, Abouzgheib W, Gajera M, Palaniswamy C, Puri N, Dellinger RP. Excessive dynamic airway collapse for the internist: new nomenclature or different entity? Postgrad Med J 2011;87(1029): 482-486.

19. Joosten S, MacDonald M, Lau KK, Bardin P, Hamilton G. Excessive dynamic airway collapse co-morbid with COPD diagnosed using 320-slice dynamic CT scanning technology. Thorax 2012;67(1):9596.

20. Murgu SD, Cherrison LJ, Colt HG. Respiratory failure due to central airway collapse. Respir Care 2007;52(6):752-754. 